\title{
Luminescent solar concentrators
}

\author{
J. S. Batchelder, A. H. Zewail, T. Cole \\ Department of Applied Physics \\ and \\ Arthur Amos Noyes Laboratory of Chemical Physics \\ California Institute of Technology, Pasadena, California 91125
}

\begin{abstract}
A type of solar concentrator for photovoltaics utilizing light pipe trapping of luminescence is described. Total collector efficiencies of $3.2 \%$ have been measured, and efficiencies of $10 \%$ appear theoretically possible. The photodegradation lifetime of the dyes presently used is about one year under optimal conditions.
\end{abstract}

\section{Introduction}

The Luminescent Solar Concentrator, or LSC, is a solar concentrator based on light pipe trapping of molecular or ionic luminescence. The conceptual operation of an LSC is illustrated by the diagram of a Planar Solar Concentrator (PSC) shown in Figure 1; a transparent material like polymethyl methacrylate

THE PLANAR SOLAR CONCENTRATOR (PSC).

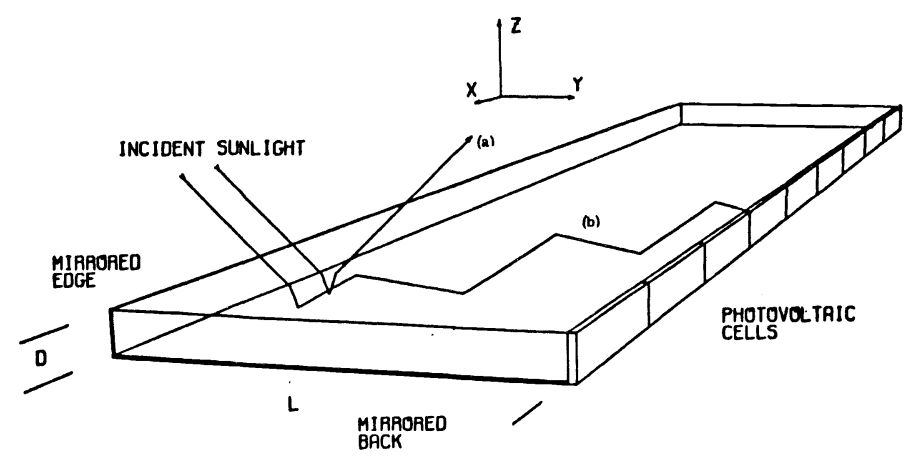

Figure 1. A planar solar concentrator, or PSC, which is a particular embodiment of an LSC. Sunlight enters from above and passes twice through the plate thickness D, the backing mirror being separated from the plate by an air gap. Part of this flux is absorbed by a dye or inorganic ion, and the ensuing luminescence can either escape back out of the face (A) or be trapped by total internal reflection (B). This trapped light will then propagate to the photovoltaic cells (PVC) where it is absorbed and converted into electricity.

(PMMA) is impregnated with guest luminescent absorbers, such as organic dye molecules having strong absorption bands in the visible and UV regions of the spectrum, and also having an efficient quantum yield of emission. Solar photons entering the upper face of the plate are absorbed, and luminescent photons are then emitted. Snell's law dictates that the probability that the luminescent photons will be trapped in the plate by total internal reflection is $\left(1-1 / \mathrm{n}^{2}\right)^{1 / 2}$, where $\mathrm{n}$ is the index of the host material. For example, about $75 \%$ of the emission is trapped in a PMMA plate with an index of 1. 49. Successive reflections transport the luminescence to the edge of the plate, where they can enter an edge-mounted array of Photovoltaic Cells, or PVCs.

\section{Theoretical performance}

An LSC's performance can be broken down into four factors: initial absorption of the solar flux, energy transfer mechanisms between the absorbing dyes or ions, transport of the energy to the edge of the plate, 
and the response of the attached cells to the concentrated light. The first LSC device reported ${ }^{1}$ contained a single type of organic laser dye in a PMMA host. Typically a single dye can absorb about $15 \%$ of the incident solar flux above the bandgap energy of silicon, so that a single dye plate has a limited efficiency. We proposed the use of many different types of dye molecules in the same plate. ${ }^{2}$ These are chosen such that the emission band of one dye strongly overlaps the absorption band of the next, allowing an excitation to cascade from one dye to the next, undergoing final emission by the reddest dye. In this way, the effective absorption bandwidth can be extended over the entire visible spectrum. Another technique to extend the effective absorption band is to use a series of parallel plates, each containing a different dye, and each plate outputting light to cells with different bandgaps. ${ }^{3}$ This effectively splits the sunlight into wavelength bands and concentrates it for the optimal cells in each band.

In a system which contains a mixture of different dyes, an excitation can be transferred from one dye to a similar dye molecule, as well as from one type of dye to another. This is possible because there is typically a significant overlap between the emission and extinction spectra of a dye, as shown in Figure 2.

\section{RHODAMINE-575 SPECTRA IN PMMA.}

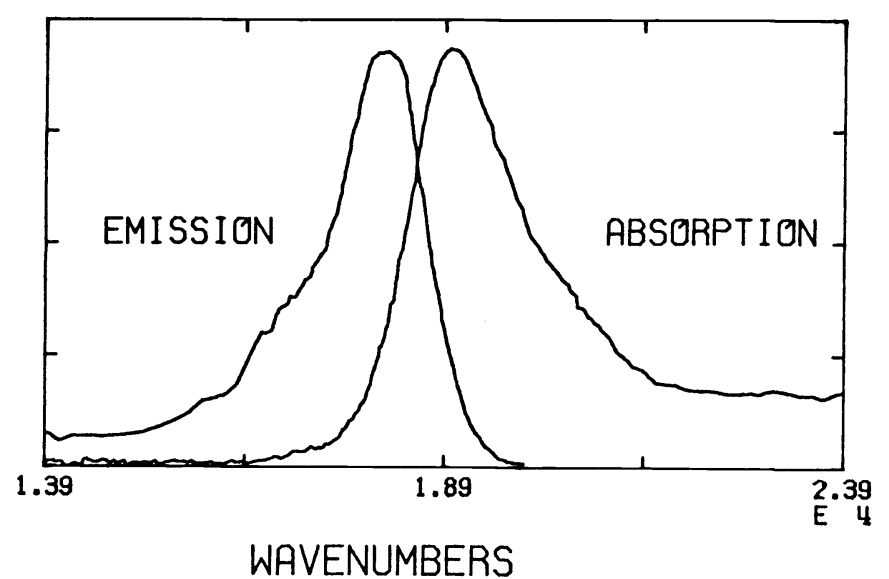

Figure 2. The absorption and emission spectra of the laser dye rhodamine-575 in PMMA. A sample was prepared with an effective dye concentration of $1.3 \mathrm{E}-5$ molar, as determined by the measured peak optical density and the known peak extinction coefficient of R-575 in methanol. The absorption was measured through a pathlength of $2 \mathrm{~mm}$, the measured peak optical density was . 27 . The emission spectrum was the surface emission due to a 20,492 wavenumber $(4880 \AA$ ) excitation.

Each time a dye molecule absorbs the emission from a similar dye molecule, or undergoes selfabsorption, there is a chance that the excitation will not cause an emission, or that the emission will not be internally trapped within the plate. For example, while the probability for trapping a single emission in a PMMA plate is $75 \%$, including the effect of self-absorption drops the probability to (. 75) $* * n s$, where $\mathrm{ns}$ is the number of self-absorptions undergone by the emission.

In a zero-order model of the performance of a LSC-PVC system, we assume that the absorbed solar flux is cascaded to the lowest energy dye, that the average number of self-absorptions by this dye is ns, and that the resulting emission is transported without loss through the plate into the cell. Under these assumptions, the collector efficiency is:

$$
\begin{aligned}
& \text { LSC Collector Efficiency }=\text { AM1 Cell Efficiency } \\
& \text { * (Dye Quantum Eff. * Trapping Eff. })^{\text {ns }} \\
& \text { * (Solar Flux above bandgap absorbed by LSC) } \\
& \text { / (Solar Flux above bandgap of the cell) }
\end{aligned}
$$

If the number of self-absorptions is assumed to be 2, and a silicon cell with $18 \%$ AM1 efficiency is attached to a multi-dye plate, then the predicted efficiency should be between $9 \%$ and $12 \%$.

\section{Observed performance}

The highest efficiency plate produced to day had a total collector efficiency of $3.2 \%{ }^{4}$ These LSCs are formed by applying thin films containing the dye to the back of an acrylic plate. A promising alternative 
is to fill the gap between two parallel glass plates with a mixture of dyes in a solvent such as ethylene glycol. We have obtained efficiences of $2 \%$ on a preliminary prototype liquid system. ${ }^{6}$

\section{Principle problems}

The outstanding questions to be answered to realize a practical power system are efficiency and lifetime. The best present devices are about a factor of three less efficient than calculations initially predicted they should be. The predominant cause of this loss appears to be a higher rate of self-absorption than was expected. Two avenues are being pursued to reduce this loss. One is to design a specialized molecule which has both a large Stoke's shift and a high quantum efficiency. ${ }^{7}$ Our approach has been to deliberately modify the environment of the dyes to narrow their absorption and emission peaks, so as to reduce their overlap. For example, in Figure 3 we show the emission spectra for rhodamine-575 in PMMA as a func-

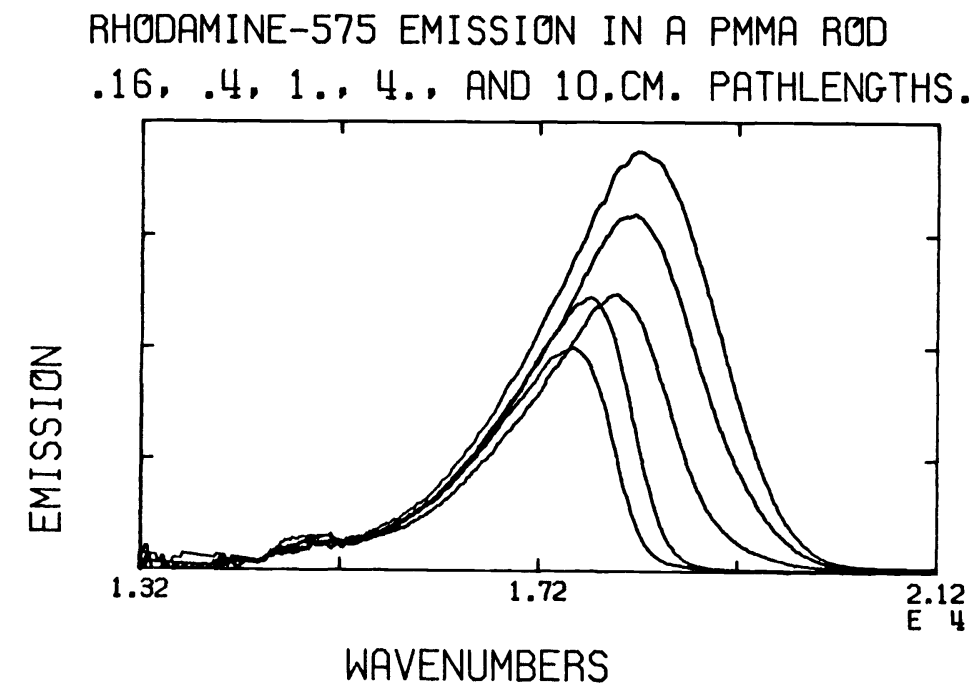

Figure 3. The emission spectra of a PMMA rod containing R-575 for five different self-absorption pathlengths. The same plastic of Figure 2 was excited by 22, 941 wavenumber (4359 $A$ ) light at five different distances from the end of the rod illuminating a spectrometer. The spectrum with a bluest emission travelled the shortest pathlength. Notice that the self-absorption effect lowers the fraction of light collected as the pathlength increases, and that for the longest pathlengths the red tail becomes stronger due to contributions from the emission of self-absorbed excitation.

tion of the pathlength that the emission travelled through the plastic. Figure 4 is a similar series of

RHODAMINE-575 EMISSION IN METHANOL

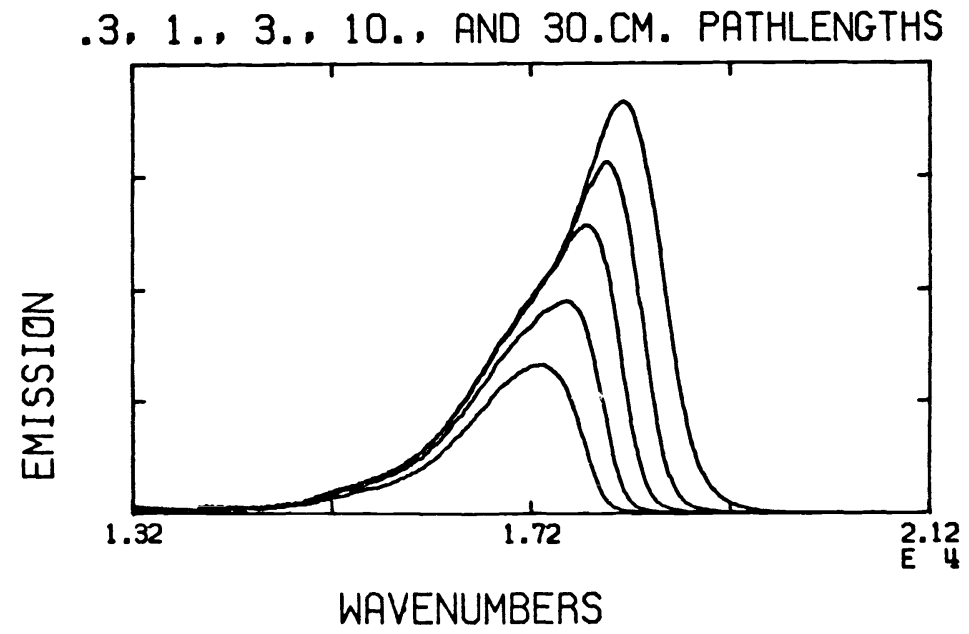


Figure 4. The emission spectra of a glass tube containing R-575 dissolved in methanol for five different pathlengths. In contrast to Figure 3 , the dye in the methanol solution is at $9.2 \mathrm{E}-5$ molar, or seven times the concentration of the dye in the plastic, but the total loss due to self-absorption is similar. While the lost flux is principly on the blue emission tail in the methanol solution, the loss is fairly uniform in the plastic, indicating that the absorption spectrum of the dye has much more broad tails in the plastic than in the liquid.

spectra of the same laser dye dissolved in methanol. The absorption tail of the dye in methanol appears to dye away faster going to lower energy than it does in the PMMA sample, and the spectra appear more peaked, implying that there is considerably more inhomogeneous broadening in the plastic sample than in the methanol. This suggests that the liquid might prove to be a more efficient system due to reduced self-absorption.

While the photodegradation half-life varies orders of magnitudes from one dye to another, typical lifetimes under solar exposure for these dyes is about a year under optimal conditions. Plastics such as cellulose acetate buterate form a more stable host than PMMA, and in general dyes in liquid solvents are more stable than they are in plastics. An alternative to laser dye molecules are inorganic ions in glass. Such systems are comparably rugged,undergoing little or no degradation, but their quantum efficiency of luminescence is typically low.

Here at Caltech we are studying the thermal and temporal properties of the dye spectra in a variety of hosts to try and increase the overall efficiency.

\section{Cost per watt}

The principle advantage of the LSC is its high geometric gain. If the plate was perfectly efficient, light incident on the edge-mounted cells would have a flux density greater than the incident solar flux by a factor which is the area of the face of the LSC divided by the area of the edge, a factor called the geometric gain. The geometric gain of a typical plate is around 100, allowing great reductions in the required area of solar cells. If a $10 \%$ system efficiency is assumed, estimates find that an environmentally protected LSC should produce power at about $\$ 0.37$ per peak watt. ${ }^{5}$

\section{Acknowledgments}

A. H. Zewail is an Alfred P. Sloan Fellow and a Camille and Henry Dreyfus Foundation teacherscholar. This work was supported in part by a contract from the U. S. Department of Energy/Solar Energy Research Institute, and in part by ARCO Solar, Inc.

\section{References}

1. Weber, W. H. , and Lambe, J. , Appl Opt. 15, 2299 (1976).

2. Swartz, B. A., Cole, T. , and Zewail, A. $\widetilde{\text { H. }}$, Opt. Lett. 1, 73 (1977).

3. Goetzberger, A. , and Greubel, W. , Appl. Phys. 14, 123 (1977).

4. Batchelder, J. S. , Zewail, A. H. , and Cole, T., Appl. Opt. 18, 3090 (1979).

5. Friedman, P. S. , Advanced Concentrator Review Meeting, Mârch 31, 1980, Solar Energy Research Institute, 1617 Cole Boulevard, Golden, Colorado 80401.

6. Zewail, A. H. , op. cit.

7. Offenhartz, P. O'D. , op. cit. 